\title{
Performance Comparison of K-means Codebook Optimization using different Clustering Techniques
}

\author{
Dr. Tanuja K. Sarode ${ }^{1}$, Nabanita Mandal ${ }^{2}$ \\ I (Associate Professor, Computer Engineering Department, Thadomal Shahani Engineering College, India) \\ ${ }^{2}$ (Lecturer, Computer Engineering Department, Thadomal Shahani Engineering College, India)
}

\begin{abstract}
Vector quantization is a compression technique which is used to compress the image data in the spatial domain. Since it is a lossy technique, so maintaining the image quality and the compression ratio is a difficult task. For this, the codebook which stores the image data should be optimally designed. In this paper, the K-means algorithm is used to optimize the codebook. We have generated the codebooks from LBG, KPE, KFCG and Random Selection Methods. It is found that the Mean Square Error reduces for every iteration and after a certain number of iterations it stops reducing because the optimal value is reached. We can say that the codebook is optimized at that point. The results show that the codebook obtained from KFCG Algorithm has a least Mean Square Error. This shows that KFCG codebook is more nearer to the optimal point and when applied with K-means algorithm gives the best optimization in comparison with other algorithms.
\end{abstract}

Keywords: Codebook Optimization, Euclidian Distance, K-means Algorithm, Mean Square Error, Vector Quantization

\section{INTRODUCTION}

With the growing popularity of the internet, the need for transmission of images has increased. But for transmitting the image quickly, high bandwidth is required. Compression of the image is one way of fast transmission. The aim behind image compression is the reduction of irrelevant and redundant data of the image in order to store or transmit the image in an efficient way. Various lossy and lossless techniques are available for compression [1]. Lossless compression techniques are suitable where each and every minute technical detail of the image is significant. Loss of a single detail would lead to transmission of an improper image. Lossy compression methods are suitable for natural images where minor loss of fidelity is allowed. One such lossy compression technique is Vector Quantization (VQ) [2]. In this technique, the image is represented in the form of vectors. VQ is a mapping function, $\mathrm{Q}$ that maps a vector in $\mathrm{K}$-dimensional vector space, $\mathrm{R}^{\mathrm{k}}$ into a finite subset of the vector space $\mathrm{W}$ containing $\mathrm{N}$ distinct vectors [3]. Hence, $\mathrm{Q}: \mathrm{R}^{\mathrm{k}} \rightarrow \mathrm{W}$.

In this technique, the amount of data contained in an image is reduced so that images can be economically transmitted. The image data is stored in a codebook. Each vector present in the codebook is called a codevector. A good codebook is very much essential for VQ. The codebook size is decided and then after applying the different techniques for codebook generation, the Mean Square Error (MSE) is obtained. The distortion obtained from the different codebook generation algorithm varies even if size of the codebook remains same. But the minimum error is not obtained. The reason is that the codevectors in the codebook may not have reached their optimal position. When the codebook is optimized, the MSE reaches a value after which it cannot be reduced further.

In literature, vector quantization has been successfully used for image compression. In Pamela C. Cosman et al's paper [4], they have given the fundamental idea of vector quantization and how it can be used for image compression. The process of VQ involves codebook design. A survey of codebook generation techniques has been done by Tzu-Chuen Lu and Ching-Yun Chang. In their paper, LBG, Enhanced LBG, Neural Network based techniques, Genetic Algorithm based techniques etc has been discussed [5]. After the codebook is generated, optimization of the codebook is done using various algorithms. H.B. Kekre and Tanuja K. Sarode has proposed K-means algorithm for optimization of codebook [6]. LBG and KFCG algorithm has been applied by them for generating the codebook and to optimize it they have used K-means algorithm. S. Vimala has done the convergence analysis of various codebook generation techniques when K-means algorithm is applied to them for optimization [7].

In this paper, our main concentration will be on optimization of the codebook. The K-means Algorithm [8] which is a clustering technique, will be used on the codebook for optimization. In this technique, initially, the codevectors are selected randomly. But the initial selection doesn't lead to optimization. So, this process is repeated for many iterations and the MSE value is calculated for each iteration. The MSE reduces in each iteration. After some iterations, the MSE value converges. This point is the optimal point and the optimized codebook is obtained. The K-means Algorithm is applied to the codebook generated by LBG Algorithm [9], 
KPE Algorithm [10], KFCG Algorithm [11] and Random Selection method. Further, the image is reconstructed back using the optimized codebook.

This paper consists of five sections. In Section II the proposed system has been discussed. Section III contains the description of the algorithms used. Section IV provides the results obtained after application of various techniques. The conclusion is given in Section V.

\section{VQ IMAGE COMPRESSION SYSTEM}

The VQ image compression system is shown in Fig. 1. The process of vector quantization consists of encoding and decoding phases.

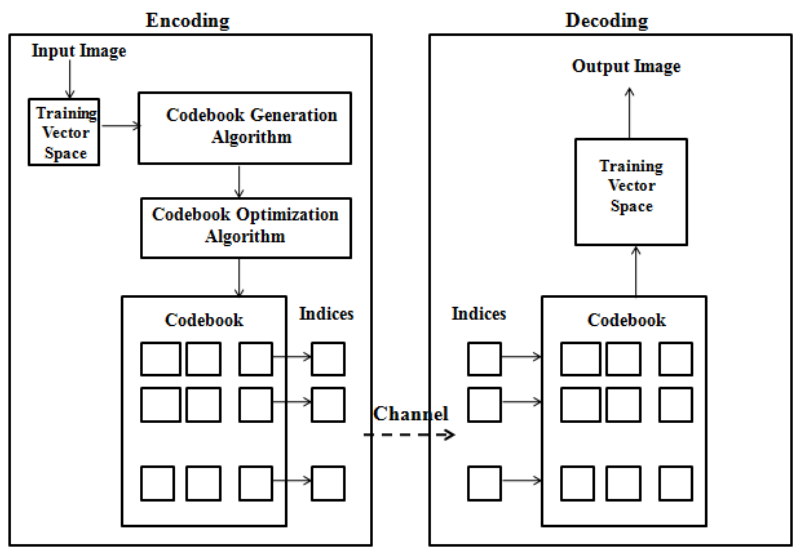

Fig. 1 VQ Image Compression System

\section{A. Encoding Phase}

The input image is converted into vectors by dividing the image matrix into $2 \times 2$ non overlapping blocks. The RGB components of each pixel are separated and values of each block are written together. This forms the codevector. The collection of all codevectors gives the training vector space of the image. From the training vector space, codebook is generated using different algorithms. The generated codebook is then optimized using the K-means algorithm. An index is created which keeps track of each codevector. The optimized codebook and the index are used to reconstruct the image.

\section{B. Decoding Phase}

In the decoding phase, the image is reconstructed back using the optimized codebook and the index. A new training vector space is created in the receiver's side which consists of the codebook values. The correct position is obtained using the index. The image is reconstructed back using this training vector space.

\section{CODEBOOK GENERATION AND OPTIMIZATION ALGORITHMS}

The various algorithms applied for the generation of codebook are LBG, KPE and KFCG. Apart from these, a new method of Random Selection has also been introduced.

\section{A. LBG Algorithm}

The LBG algorithm has been introduced by Linde-Buzo-Gray [9]. First the training vector space is created and the centroid is obtained. The centroid is considered as the first codevector. Now, constant error is added to the codevector and two new vectors are obtained. Fig.2 shows the LBG clustering. v1 and v2 are the new generated codevectors. The Euclidean distance between the training vector space and the vectors v1 and $\mathrm{v} 2$ is computed. After computing the Euclidean distance, the training vector which is nearer to v1 is put in one cluster and the training vector which is nearer to v2 is put in another cluster. Two clusters are obtained in the first iteration. In the next iteration, this procedure is repeated for both the clusters.

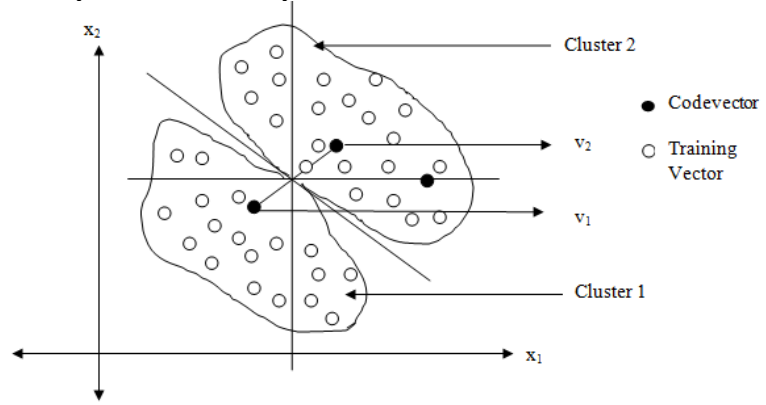

Fig. 2 LBG Clustering 
The steps of LBG algorithm are as follows:

1. Generate the training vector space, $\mathrm{T}$ of the image which contains $\mathrm{M}$ training vectors.

$\mathrm{T}=\left\{\mathrm{X}_{1}, \mathrm{X}_{2}, \mathrm{X}_{3} \ldots \mathrm{X}_{\mathrm{M}}\right\}$

$\mathrm{X}_{\mathrm{i}}$ is the training vector which is represented as $\mathrm{X}_{\mathrm{i}}=\left\{\mathrm{x}_{\mathrm{i} 1}, \mathrm{x}_{\mathrm{i} 2}, \mathrm{x}_{\mathrm{i} 3}, \ldots \mathrm{X}_{\mathrm{ik}}\right\}$

where,

$\mathrm{k}$ denotes the dimension.

2. Find Centroid, $\mathrm{C}$ of the training vector space by taking the average of each column. This centroid is the first codevector.

$\mathrm{C}=\left\{\mathrm{C}_{1}, \mathrm{C}_{2}, \mathrm{C}_{3} \ldots \mathrm{C}_{\mathrm{M}}\right\}$

3. Two new vectors are obtained after adding constant error, $\mathrm{E}$ to the codevector.

$\mathrm{C} 1=\mathrm{C}+\mathrm{E}$ and $\mathrm{C} 2=\mathrm{C}-\mathrm{E}$

4. Find the Euclidian distance of the training vector space with these two vectors.

$\mathrm{D}\left(\mathrm{X}_{\mathrm{i}}-\mathrm{C}_{\mathrm{j}}\right)==\sum_{\mathrm{p}=1}^{\mathrm{k}}\left(\mathrm{x}_{\mathrm{ip}}-\mathrm{c}_{\mathrm{jp}}\right)^{2}$

where,

$\mathrm{X}_{\mathrm{i}}$ is the training vector,

$\mathrm{C}_{\mathrm{j}}$ is the codevector

5. Put the training vector in first cluster if the Euclidian distance between the training vector and the codevector $\mathrm{C} 1$ is less else put the training vector in the second cluster.

6. Repeat the steps 2 to 5 for every cluster.

7. Stop when desired codebook size is obtained.

\section{B. KPE Algorithm}

Kekre's Proportionate Error (KPE) algorithm [10] uses proportionate error instead of constant error. The training vector space is created and the centroid is obtained. The centroid is the first codevector. This proportionate error is added to the codevector and two new vectors are obtained. The proportionate error is obtained as follows:

- Consider the codevector $\mathrm{C}$ and Error Vector $\mathrm{E}$, which is represented as $\mathrm{C}=\left\{\mathrm{c}_{1}, \mathrm{c}_{2}, \mathrm{c}_{3}, \ldots . \mathrm{c}_{\mathrm{k}}\right\}$ and

- $\mathrm{E}=\left\{\mathrm{e}_{1}, \mathrm{e}_{2}, \mathrm{e}_{3, \ldots \ldots} \mathrm{e}_{\mathrm{k}}\right\}$ respectively.

- $\quad$ Find $c_{j}$ where $c_{j}=\min \left\{c_{i} / i\right\}$ and value of $i$ varies from 1 to $\mathrm{k}$.

$\mathrm{j}$ is the index of the vector member whose value is minimum among the other vector members.

- Assign $\mathrm{e}_{\mathrm{j}}=1$

- If $\mathrm{c}_{\mathrm{i}} / \mathrm{c}_{\mathrm{j}} \leq 10$ then assign $\mathrm{e}_{\mathrm{i}}=\mathrm{c}_{\mathrm{i}} / \mathrm{c}_{\mathrm{j}}$

- Else assign $e_{i}=10$ where, $i \neq j$ and value of $i$ varies from 1 to $\mathrm{k}$.

The Euclidean distance between the training vector space and the new vectors is computed. The training vector which is nearer to first vector is put in one cluster and the training vector which is nearer to second vector is put in another cluster. Two clusters are obtained in the first iteration. Thus, the size of the codebook is increased by two. In the next iteration, this procedure is repeated for both the clusters. The codebook size becomes four. This procedure is repeated till the codebook size becomes equal to the size specified by the user.

\section{KFCG Algorithm}

Kekre's Fast Codebook Generation (KFCG) algorithm [11] does not use Euclidean distance for comparison and also no error is added to get the new vectors. So, the codebook generation is faster as compared to other algorithms . The training vector space is considered as a single cluster initially. The centroid obtained is the codevctor. Fig. 3 shows the $1^{\text {st }}$ iteration of KFCG Clustering. $\mathrm{C} 1$ is the codevector obtained after centroid calculation. The first member of training vector is compared to the first member of codevector in the first iteration. The training vector is put in first cluster if its first member is less than the first member of codevector. Otherwise the training vector is put in the second cluster. In the second iteration, division of the first cluster is done by comparing second member of the training vector with the second member of codevector. Fig. 4 shows $2^{\text {nd }}$ iteration of KFCG Clustering. Again second cluster is divided into two clusters by comparing the second member of the training vector with that of the codevector. This process is repeated till desired codebook size is reached. 


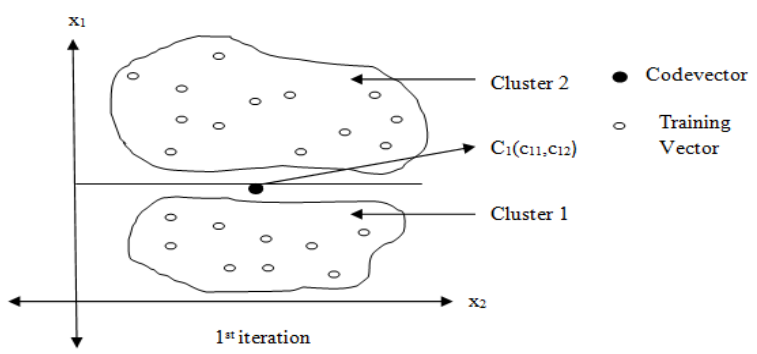

Fig. $31^{\text {st }}$ iteration of KFCG Clustering

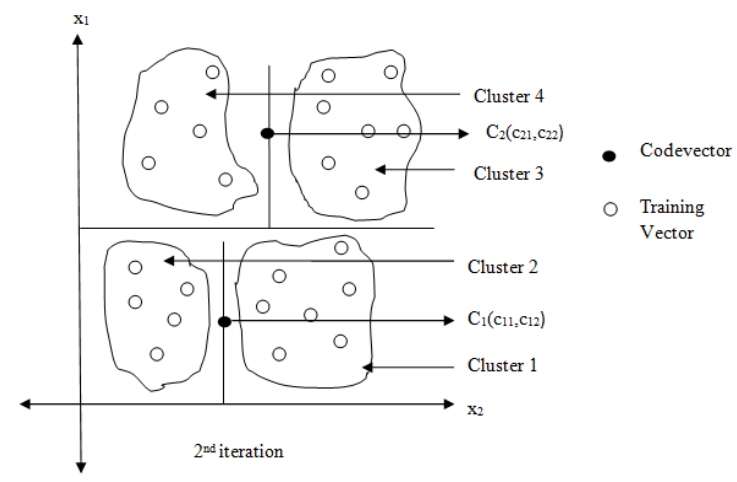

Fig. $42^{\text {nd }}$ iteration of KFCG Clustering

A new method of Random Selection has been introduced in this paper for generating the codebook.

\section{RANDOM SELECTION METHOD}

In this method, codebook of desired size is obtained by random selection of vectors from the training vector space. In other words it can be said that the size of the codebook is dependent on the number of random vectors initially chosen.

The steps are as follows:-

1. Generate the training vector space, $\mathrm{T}$ of the image which contains $\mathrm{M}$ training vectors.

$\mathrm{T}=\left\{\mathrm{X}_{1}, \mathrm{X}_{2}, \mathrm{X}_{3} \ldots \mathrm{X}_{\mathrm{M}}\right\}$

$\mathrm{X}_{\mathrm{i}}$ is the training vector and it is represented as $\mathrm{X}_{\mathrm{i}}=\left\{\mathrm{x}_{\mathrm{i} 1}, \mathrm{x}_{\mathrm{i} 2}, \mathrm{x}_{\mathrm{i} 3}, \ldots \mathrm{x}_{\mathrm{ik}}\right\}$

where,

$\mathrm{k}$ denotes the dimension.

2. Select $\mathrm{K}$ random vectors from the training vector space where $\mathrm{K}$ denotes the desired codebook size. These initial random vectors serve the purpose of the initial codebook.

The codebook obtained by LBG algorithm, KPE algorithm, KFCG algorithm and Random Selection Method are fed as input to the K-means algorithm for optimization.

\section{E. K-means Algorithm}

The K-means algorithm [9] is a clustering algorithm where $\mathrm{K}$ denotes the size of the cluster. The basic idea behind this algorithm is to form $\mathrm{K}$ clusters and assign each object to one of the $\mathrm{K}$ clusters in such a way that the measure of dispersion within the cluster is minimized. The dispersion can be measured using the squared Euclidean distance. This algorithm aims at minimizing the objective function. Here, the mean square error is the objective function. So, the MSE reduces at each iteration and after the codebook is optimized, the MSE reaches a value after which it stops reducing. It converges at the optimal point.

In this algorithm, K-random vectors are selected from the training vector space and call it as codevectors. The squared Euclidean distance of all the training vectors with the selected $\mathrm{K}$ vectors are obtained and $\mathrm{K}$ clusters are formed.

If the squared Euclidean distance of training vector $X_{j}$ with $i^{\text {th }}$ codevector is minimum then $X_{j}$ is put in $\mathrm{i}^{\text {th }}$ cluster. Centroid of each cluster is obtained. The centroids of all clusters obtained in the previous iteration form the set of new codevectors which is the input to K-Means algorithm for the next iteration. The MSE for each of the K clusters is computed and net MSE is obtained. This process is repeated till the net MSE converges. The MSE is calculated using the following formula:- 


$$
\operatorname{MSE}(\mathrm{m})=\frac{1}{\mathrm{Zk}} \sum_{\mathrm{r}=1}^{\mathrm{Z}}\left(\mathrm{X}_{\mathrm{r}}-\mathrm{C}_{\mathrm{m}}\right)^{2}
$$

where,

$\mathrm{m}$ is the cluster number,

$\mathrm{Z}$ is the number of vectors in that cluster,

$\mathrm{k}$ is the dimension,

$\mathrm{X}_{\mathrm{r}}$ is $\mathrm{r}^{\text {th }}$ training vector,

$\mathrm{C}_{\mathrm{m}}$ is the codevector of $\mathrm{m}^{\text {th }}$ cluster

Instead of selecting $\mathrm{K}$ random vectors, the codebooks of size K obtained by LBG, KPE and KFCG, Random Selection Method [12] are used for optimization using the K-means algorithm.

The steps are as follows:-

1. Generate the training vector space, $\mathrm{T}$ of the image which contains $\mathrm{M}$ training vectors.

$\mathrm{T}=\left\{\mathrm{X}_{1}, \mathrm{X}_{2}, \mathrm{X}_{3} \ldots \mathrm{X}_{\mathrm{M}}\right\}$

$\mathrm{X}_{\mathrm{i}}$ is the training vector and it is represented as $\mathrm{X}_{\mathrm{i}}=\left\{\mathrm{x}_{\mathrm{i} 1}, \mathrm{x}_{\mathrm{i} 2}, \mathrm{x}_{\mathrm{i} 3}, \ldots \mathrm{x}_{\mathrm{ik}}\right\}$

where,

$\mathrm{k}$ denotes the dimension.

2. Generate codebook containing K codevectors using LBG, KPE, KFCG or Random Selection Method where $\mathrm{K}$ is the desired codebook size.

3. Find the squared Euclidean distance of all the training vectors with the $\mathrm{K}$ codevectors and $\mathrm{K}$ clusters are formed.

$\mathrm{D}\left(\mathrm{X}_{\mathrm{i}}-\mathrm{C}_{\mathrm{j}}\right)=\sum_{\mathrm{p}=1}^{\mathrm{k}}\left(\mathrm{x}_{\mathrm{ip}}-\mathrm{c}_{\mathrm{jp}}\right)^{2}$

where,

$\mathrm{X}_{\mathrm{i}}$ is the training vector,

$\mathrm{C}_{\mathrm{j}}$ is the codevector.

4. If the squared Euclidean distance of the $X_{j}$ with $i^{\text {th }}$ codevector is minimum then put $X_{j}$ in $i^{\text {th }}$ cluster.

If the squared Euclidean distance of $X_{j}$ with codevectors happens to be minimum for more than one codevector then put $X_{j}$ in any one of them.

5. Compute the centroid for each cluster.

6. Find MSE for all the $\mathrm{K}$ clusters.

7. Calculate net MSE.

8. Replace the initial codevectors by the centroids of each cluster.

9. Repeat steps 3 to 5 till the two successive net MSE values are same.

\section{RESULTS}

The techniques are implemented on Intel Core i5-3210M, $2.50 \mathrm{GHz}, 4 \mathrm{~GB}$ RAM machine and Matlab $\mathrm{R} 2011 \mathrm{~b}$ is used. The algorithms are applied on ten color images each having size of $256 \times 256 \times 3$.

Training Images of size $256 \times 256 \times 3$ are shown in Fig. 5 .

The mean square error and the number of iterations required for optimization of codebook obtained from LBG, KPE, KFCG algorithms and Random Selection Method on codebook size 128, 256, 512 and 1024 are shown in Table I, II, III and IV respectively.

The sample initial and final images of Peacock for LBG, KPE, KFCG and Random Selection Methods with codebook size 256 are shown in Fig. 6.

The Variation of MSE with Number of Iterations for Peacock image for codebook size 256 is shown in

Fig. 7. 


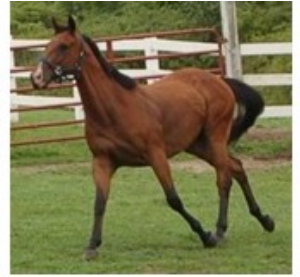

(a)

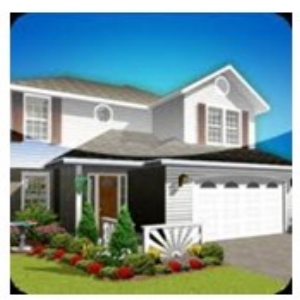

(e)

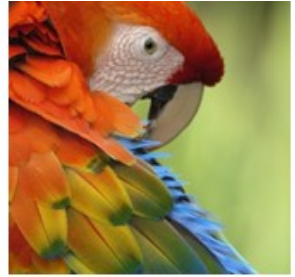

(b)

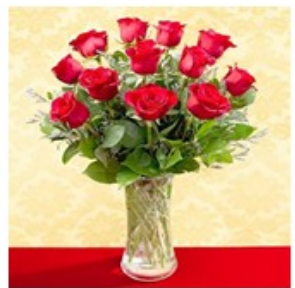

(f)

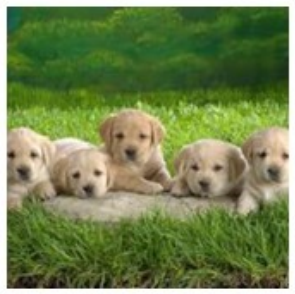

(i)

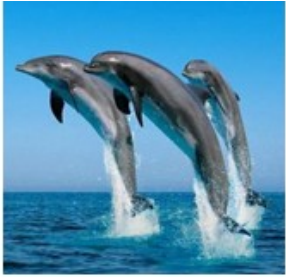

(c)

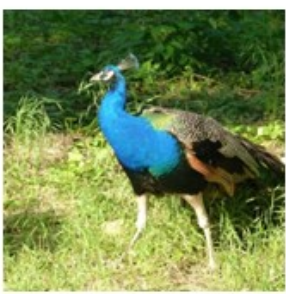

(g)

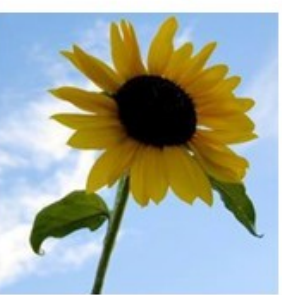

(j)

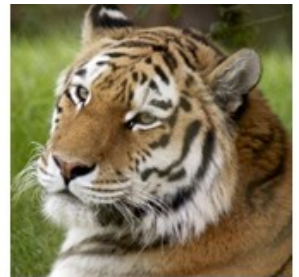

(d)

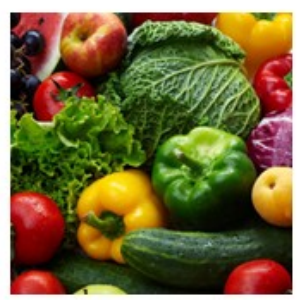

(h)

Fig. 5 Training Images (a) Horse (b) Macaw (c) Dolphins (d) Tiger (e) House (f) Roses (g) Peacock (h) Vegetables (i) Puppies (j) Sunflower

Table I. Result of K-means Algorithm on codebook size 128

\begin{tabular}{|c|c|c|c|c|c|}
\hline S. no. & Image & Algorithm & Initial MSE & Final MSE & No. of iterations \\
\hline \multirow{4}{*}{1.} & \multirow{4}{*}{ Horse } & LBG & 143.74 & 64.25 & 104 \\
\hline & & KPE & 144.64 & 63.84 & 87 \\
\hline & & KFCG & 194.61 & 53.03 & 179 \\
\hline & & RANDOM & 188.59 & 69.51 & 204 \\
\hline \multirow{4}{*}{2.} & \multirow{4}{*}{ Macaw } & LBG & 259.41 & 90.19 & 237 \\
\hline & & KPE & 225.04 & 89.35 & 176 \\
\hline & & KFCG & 398.26 & 94.83 & 409 \\
\hline & & RANDOM & 273.82 & 94.06 & 122 \\
\hline \multirow{4}{*}{3.} & \multirow{4}{*}{ Dolphins } & LBG & 178.24 & 86.38 & 97 \\
\hline & & KPE & 163.07 & 87.49 & 63 \\
\hline & & KFCG & 244.63 & 59.69 & 122 \\
\hline & & RANDOM & 282.48 & 112.23 & 121 \\
\hline \multirow{4}{*}{4.} & \multirow{4}{*}{ Tiger } & LBG & 247.27 & 125.18 & 87 \\
\hline & & KPE & 252.22 & 124.29 & 109 \\
\hline & & KFCG & 206.11 & 67.43 & 90 \\
\hline & & RANDOM & 256.18 & 138.38 & 203 \\
\hline \multirow{4}{*}{5.} & \multirow{4}{*}{ House } & LBG & 274.22 & 102.11 & 112 \\
\hline & & KPE & 219.31 & 97.11 & 76 \\
\hline & & KFCG & 246.41 & 74.04 & 256 \\
\hline & & RANDOM & 275.16 & 129.93 & 192 \\
\hline \multirow{4}{*}{6.} & \multirow{4}{*}{ Roses } & LBG & 356.11 & 171.11 & 116 \\
\hline & & KPE & 319.22 & 168.15 & 118 \\
\hline & & KFCG & 533.34 & 127.15 & 148 \\
\hline & & RANDOM & 334.85 & 180.45 & 158 \\
\hline \multirow{4}{*}{7.} & \multirow{4}{*}{ Sunflower } & LBG & 115.94 & 55.13 & 92 \\
\hline & & $\mathrm{KPE}$ & 119.22 & 54.57 & 71 \\
\hline & & KFCG & 167.79 & 45.79 & 278 \\
\hline & & RANDOM & 168.18 & 65.98 & 141 \\
\hline
\end{tabular}


Performance Comparison of K-means Codebook Optimization using different Clustering

\begin{tabular}{|c|c|c|c|c|c|}
\hline & & & & & \\
\hline \multirow{4}{*}{8.} & \multirow{4}{*}{ Vegetables } & LBG & 529.29 & 224.67 & 157 \\
\hline & & KPE & 561.27 & 228.97 & 170 \\
\hline & & KFCG & 704.93 & 152.15 & 253 \\
\hline & & RANDOM & 575.58 & 233.85 & 239 \\
\hline \multirow{4}{*}{9.} & \multirow{4}{*}{ Puppies } & LBG & 141.64 & 78.73 & 73 \\
\hline & & KPE & 140.29 & 78.45 & 102 \\
\hline & & KFCG & 179.49 & 56.03 & 141 \\
\hline & & RANDOM & 186.01 & 87.12 & 232 \\
\hline \multirow{4}{*}{10.} & \multirow{4}{*}{ Peacock } & LBG & 386.11 & 207.71 & 98 \\
\hline & & KPE & 370.94 & 205.91 & 112 \\
\hline & & KFCG & 447.14 & 156.77 & 270 \\
\hline & & RANDOM & 432.55 & 223.96 & 275 \\
\hline
\end{tabular}

Table II. Result of K-means Algorithm on codebook size 256

\begin{tabular}{|c|c|c|c|c|c|}
\hline S. no. & Image & Algorithm & Initial MSE & Final MSE & No. of Iterations \\
\hline \multirow{4}{*}{1.} & \multirow{4}{*}{ Horse } & LBG & 166.41 & 56.44 & 132 \\
\hline & & $\mathrm{KPE}$ & 170.75 & 56.04 & 188 \\
\hline & & KFCG & 179.16 & 45.99 & 133 \\
\hline & & RANDOM & 138.14 & 56.97 & 234 \\
\hline \multirow{4}{*}{2.} & \multirow{4}{*}{ Macaw } & LBG & 285.53 & 85.45 & 130 \\
\hline & & KPE & 232.59 & 82.46 & 139 \\
\hline & & KFCG & 384.51 & 82.32 & 412 \\
\hline & & RANDOM & 494.82 & 72.31 & 276 \\
\hline \multirow{4}{*}{3.} & \multirow{4}{*}{ Dolphins } & LBG & 166.15 & 74.21 & 51 \\
\hline & & $\mathrm{KPE}$ & 155.57 & 76.22 & 66 \\
\hline & & KFCG & 220.66 & 62.81 & 113 \\
\hline & & RANDOM & 979.32 & 118.64 & 350 \\
\hline \multirow{4}{*}{4.} & \multirow{4}{*}{ Tiger } & LBG & 245.07 & 109.83 & 141 \\
\hline & & $\mathrm{KPE}$ & 235.12 & 109.55 & 149 \\
\hline & & KFCG & 244.54 & 78.08 & 156 \\
\hline & & RANDOM & 264.77 & 114.96 & 317 \\
\hline \multirow{4}{*}{5.} & \multirow{4}{*}{ House } & LBG & 313.73 & 105.95 & 174 \\
\hline & & KPE & 297.59 & 105.29 & 187 \\
\hline & & KFCG & 259.81 & 85.99 & 149 \\
\hline & & RANDOM & 645.79 & 141.06 & 330 \\
\hline \multirow{4}{*}{6.} & \multirow{4}{*}{ Roses } & LBG & 392.79 & 162.63 & 135 \\
\hline & & KPE & 334.15 & 163.49 & 143 \\
\hline & & KFCG & 439.23 & 131.69 & 149 \\
\hline & & RANDOM & 621.99 & 167.02 & 136 \\
\hline \multirow{4}{*}{7.} & \multirow{4}{*}{ Sunflower } & LBG & 119.78 & 44.72 & 214 \\
\hline & & KPE & 100.83 & 46.99 & 137 \\
\hline & & KFCG & 192.81 & 45.97 & 283 \\
\hline & & RANDOM & 536.71 & 55.04 & 237 \\
\hline \multirow{4}{*}{8.} & \multirow{4}{*}{ Vegetables } & LBG & 569.96 & 212.51 & 549 \\
\hline & & KPE & 537.41 & 215.89 & 340 \\
\hline & & KFCG & 520.89 & 159.79 & 306 \\
\hline & & RANDOM & 344.99 & 189.27 & 168 \\
\hline \multirow{4}{*}{9.} & \multirow{4}{*}{ Puppies } & LBG & 148.35 & 76.56 & 111 \\
\hline & & KPE & 152.19 & 74.56 & 99 \\
\hline & & KFCG & 185.71 & 58.07 & 175 \\
\hline & & RANDOM & 659.72 & 76.53 & 207 \\
\hline \multirow{4}{*}{10.} & \multirow{4}{*}{ Peacock } & LBG & 483.52 & 212.18 & 97 \\
\hline & & $\mathrm{KPE}$ & 390.01 & 205.14 & 161 \\
\hline & & KFCG & 535.95 & 165.71 & 293 \\
\hline & & RANDOM & 504.49 & 204.71 & 180 \\
\hline
\end{tabular}

Table III. Result of K-means Algorithm on codebook size 512

\begin{tabular}{|c|c|c|c|c|c|}
\hline S. no. & \multirow{2}{*}{ Image } & Algorithm & Initial MSE & Final MSE & No. of Iterations \\
\hline \multirow{3}{*}{1.} & \multirow{3}{*}{ Horse } & LBG & 151.58 & 49.26 & 146 \\
\cline { 3 - 6 } & & KPE & 146.04 & 49.59 & 114 \\
\cline { 3 - 6 } & & KFCG & 176.32 & 42.54 & 275 \\
\cline { 3 - 6 } 2. & \multirow{3}{*}{ Macaw } & RANDOM & 114.39 & 44.42 & 143 \\
& & LBG & 223.45 & 74.14 & 164 \\
\cline { 3 - 6 } & & KPE & 211.31 & 74.63 & 101 \\
\cline { 3 - 6 } & & KFCG & 358.09 & 73.24 & 252 \\
\end{tabular}


Performance Comparison of K-means Codebook Optimization using different Clustering

\begin{tabular}{|c|c|c|c|c|c|}
\hline & & & & & \\
\hline \multirow{4}{*}{3.} & \multirow{4}{*}{ Dolphins } & LBG & 166.17 & 64.39 & 64 \\
\hline & & KPE & 146.63 & 67.61 & 57 \\
\hline & & KFCG & 244.63 & 59.69 & 122 \\
\hline & & RANDOM & 285.76 & 106.32 & 170 \\
\hline \multirow{4}{*}{4.} & \multirow{4}{*}{ Tiger } & LBG & 227.58 & 95.91 & 168 \\
\hline & & KPE & 220.83 & 95.61 & 95 \\
\hline & & KFCG & 206.11 & 67.43 & 90 \\
\hline & & RANDOM & 287.95 & 101.91 & 245 \\
\hline \multirow{4}{*}{5.} & \multirow{4}{*}{ House } & LBG & 285.06 & 94.17 & 161 \\
\hline & & KPE & 233.02 & 99.95 & 128 \\
\hline & & KFCG & 246.41 & 74.04 & 256 \\
\hline & & RANDOM & 282.61 & 118.17 & 191 \\
\hline \multirow{4}{*}{6.} & \multirow{4}{*}{ Roses } & LBG & 338.45 & 154.88 & 196 \\
\hline & & KPE & 300.37 & 148.93 & 148 \\
\hline & & KFCG & 533.34 & 127.15 & 148 \\
\hline & & RANDOM & 350.39 & 144.72 & 116 \\
\hline \multirow{4}{*}{7.} & \multirow{4}{*}{ Sunflower } & LBG & 120.66 & 42.31 & 247 \\
\hline & & KPE & 89.34 & 40.43 & 117 \\
\hline & & KFCG & 167.79 & 45.79 & 278 \\
\hline & & RANDOM & 364.33 & 46.89 & 199 \\
\hline \multirow{4}{*}{8.} & \multirow{4}{*}{ Vegetables } & LBG & 514.36 & 205.35 & 277 \\
\hline & & $\mathrm{KPE}$ & 466.29 & 201.33 & 211 \\
\hline & & KFCG & 704.93 & 152.15 & 253 \\
\hline & & RANDOM & 271.29 & 158.78 & 138 \\
\hline \multirow{4}{*}{9.} & \multirow{4}{*}{ Puppies } & LBG & 137.28 & 67.92 & 102 \\
\hline & & KPE & 139.32 & 68.12 & 114 \\
\hline & & KFCG & 179.49 & 56.03 & 141 \\
\hline & & RANDOM & 642.68 & 69.26 & 336 \\
\hline \multirow{4}{*}{10.} & \multirow{4}{*}{ Peacock } & LBG & 397.88 & 187.98 & 117 \\
\hline & & KPE & 346.79 & 184.44 & 120 \\
\hline & & KFCG & 447.14 & 156.77 & 270 \\
\hline & & RANDOM & 454.69 & 187.27 & 151 \\
\hline
\end{tabular}

Table IV. Result of K-means Algorithm on codebook size 1024

\begin{tabular}{|c|c|c|c|c|c|}
\hline S. no. & Image & Algorithm & Initial MSE & Final MSE & No. of Iterations \\
\hline \multirow{4}{*}{1.} & \multirow{4}{*}{ Horse } & LBG & 138.01 & 45.27 & 82 \\
\hline & & KPE & 136.51 & 46.36 & 92 \\
\hline & & KFCG & 169.39 & 39.01 & 152 \\
\hline & & RANDOM & 247.12 & 103.64 & 54 \\
\hline \multirow{4}{*}{2.} & \multirow{4}{*}{ Macaw } & LBG & 231.65 & 66.81 & 152 \\
\hline & & KPE & 182.75 & 68.07 & 118 \\
\hline & & KFCG & 374.99 & & \\
\hline & & RANDOM & 862.83 & 152.07 & 216 \\
\hline \multirow{4}{*}{3.} & \multirow{4}{*}{ Dolphins } & LBG & 156.72 & 57.06 & 70 \\
\hline & & $\mathrm{KPE}$ & 136.51 & 60.27 & 51 \\
\hline & & KFCG & 325.59 & 57.04 & 111 \\
\hline & & RANDOM & 950.55 & 124.34 & 286 \\
\hline \multirow{4}{*}{4.} & \multirow{4}{*}{ Tiger } & LBG & 212.61 & 88.11 & 104 \\
\hline & & KPE & 212.31 & 87.18 & 109 \\
\hline & & KFCG & 172.34 & 54.95 & 66 \\
\hline & & RANDOM & 232.43 & 94.57 & 176 \\
\hline \multirow{4}{*}{5.} & \multirow{4}{*}{ House } & LBG & 245.54 & 83.44 & 172 \\
\hline & & KPE & 209.25 & 89.02 & 222 \\
\hline & & KFCG & 231.46 & 81.22 & 227 \\
\hline & & RANDOM & 257.95 & 98.54 & 250 \\
\hline \multirow{4}{*}{6.} & \multirow{4}{*}{ Roses } & LBG & 318.04 & 142.85 & 167 \\
\hline & & KPE & 272.03 & 144.56 & 153 \\
\hline & & KFCG & 571.35 & 122.12 & 127 \\
\hline & & RANDOM & 519.31 & 153.84 & 173 \\
\hline \multirow{4}{*}{7.} & \multirow{4}{*}{ Sunflower } & LBG & 111.32 & 40.67 & 137 \\
\hline & & KPE & 78.29 & 34.87 & 91 \\
\hline & & KFCG & 205.23 & 46.21 & 132 \\
\hline & & RANDOM & 581.69 & 43.76 & 193 \\
\hline \multirow{4}{*}{8.} & \multirow{4}{*}{ Vegetables } & LBG & 470.38 & 195.76 & 197 \\
\hline & & KPE & 431.91 & 194.56 & 275 \\
\hline & & KFCG & 507.17 & 153.23 & 311 \\
\hline & & RANDOM & 564.21 & 363.09 & 117 \\
\hline \multirow[b]{2}{*}{9.} & \multirow[b]{2}{*}{ Puppies } & LBG & 136.37 & 62.91 & 104 \\
\hline & & KPE & 137.48 & 63.81 & 128 \\
\hline
\end{tabular}


Performance Comparison of K-means Codebook Optimization using different Clustering

\begin{tabular}{|c|c|c|c|c|c|}
\hline & KFCG & 168.21 & 51.54 & 205 \\
\cline { 3 - 6 } & & RANDOM & 607.13 & 62.62 & 244 \\
\hline \multirow{3}{*}{10.} & \multirow{3}{*}{ Peacock } & LBG & 368.88 & 172.84 & 148 \\
\cline { 3 - 6 } & & KPE & 318.03 & 170.15 & 123 \\
\cline { 3 - 6 } & & KFCG & 586.07 & 153.54 & 205 \\
\cline { 3 - 6 } & & RANDOM & 364.42 & 168.45 & 128 \\
\hline
\end{tabular}

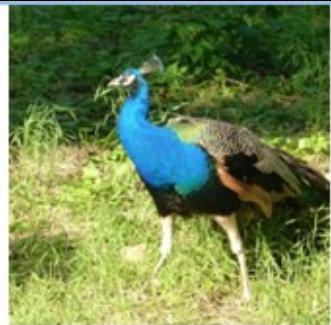

(a)

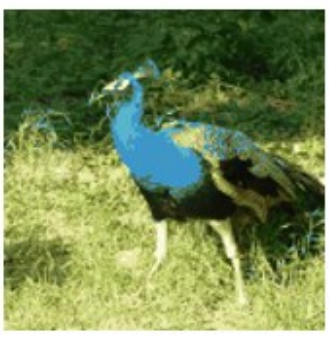

(b)

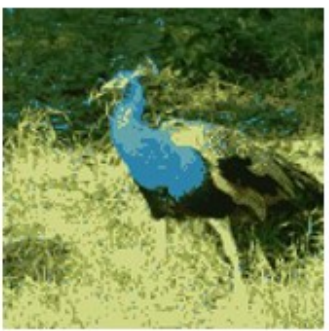

(f)

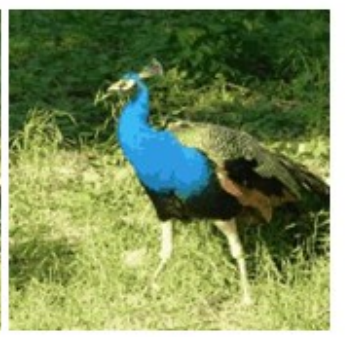

(c)

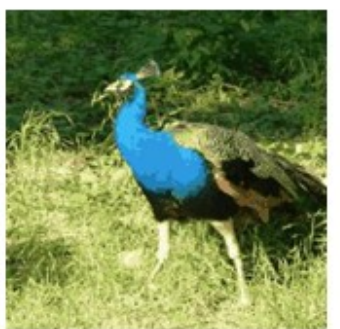

(g)

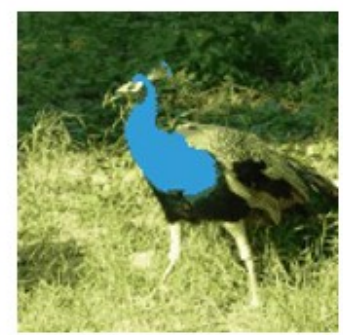

(d)

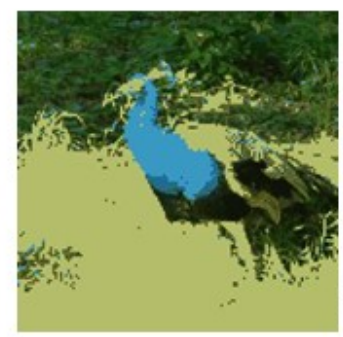

(h)

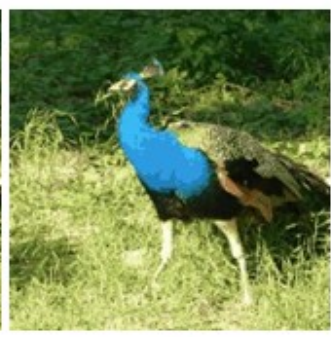

(e)

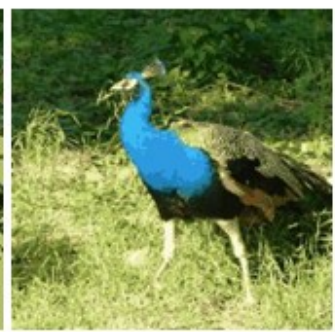

(i)

Fig. 6 Initial and Final Images (a) Original Image (b) Initial image for LBG with MSE $=483.52$ (c) Final image with MSE $=212.18$ (d) Initial image for $\mathrm{KPE}$ with $\mathrm{MSE}=\mathbf{3 9 0 . 0 1}$ (e) Final image with $\mathrm{MSE}=\mathbf{2 0 5 . 1 4}$ (f) Initial image for KFCG with MSE=535.95 (g) Final image with MSE=165.71 (h) Initial image for Random Selection with MSE $=504.49$ (i) Final image with $\mathrm{MSE}=204.71$

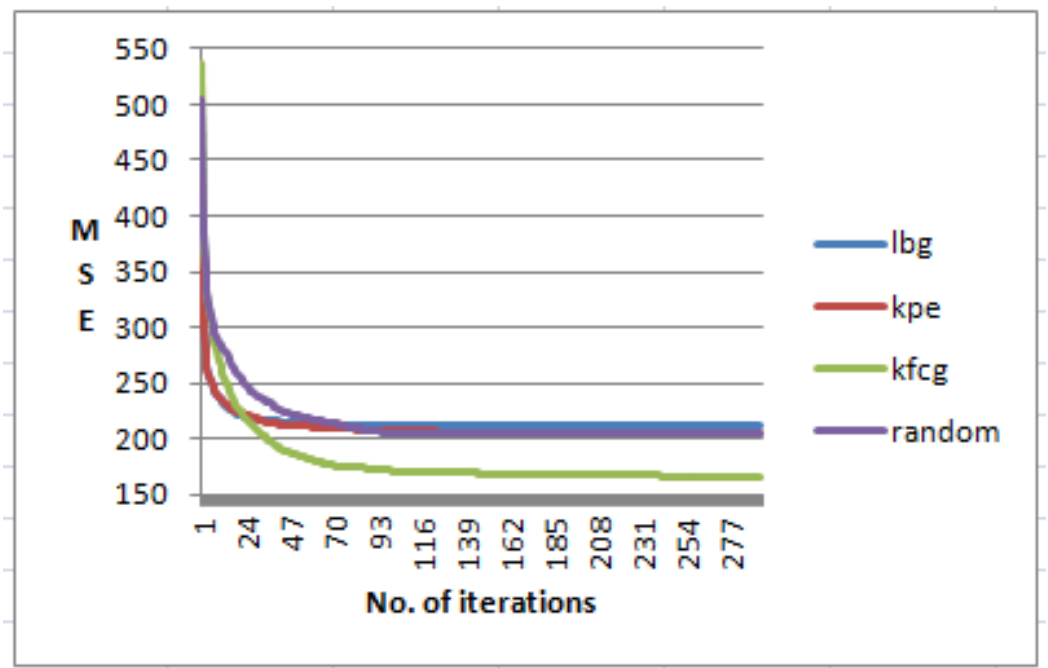

Fig. 7 Variation of MSE with Number of Iterations for Peacock image for codebook size 256 


\section{CONCLUSION}

Codebook generation and optimization can be done using various algorithms. An optimized codebook gives the proper reconstructed image which has less mean square error when compared with the original image. In this paper, codebook is generated using LBG, KPE, KFCG and Random Selection Method. The K-means algorithm is used on the generated codebook for optimization. We have used different images to show the optimization. The codebook size is varied to show the change in mean square error. It is observed that, in most of the cases, the codebook obtained from KFCG algorithm gives less mean square error in comparison to other algorithms. So, when the K-means algorithm is applied to the codebook obtained from KFCG algorithm, the best optimized codebook is obtained .

\section{REFERENCES}

[1] R. Navaneethakrishnan, "Study of Image Compression Techniques," International Journal of Scientific \& Engineering Research, Vol. 3, No. 7, pp. 1-5, July 2012.

[2] G. Boopathy and S. Arockiasam, "Implementation of Vector Quantization for Image Compression - A Survey," Global Journal of Computer Science and Technology, Vol. 10, No. 3, pp. 22-28, April 2010.

[3] Carlos R.B. Azevedo, Esdras L. Bispo Junior, Tiago A. E. Ferreira, Francisco Madeiro, and Marcelo S. Alencar, “An Evolutionary Approach for Vector Quantization Codebook Optimization,” Springer-Verlag Heidelberg, pp. 452-461, 2008.

[4] Pamela C. Cosman, Karen L. Oehler, Eve A. Riskin, and Robert M. Gray, "Using Vector Quantization for Image Processing," In Proc. Of The IEEE, Vol. 81, No. 9, pp. 1326-1341, September 1993.

[5] Tzu-Chuen Lu and Ching-Yun Chang, "A Survey of VQ Codebook Generation," Journal of Information Hiding and Multimedia Signal Processing, Vol. 1, No. 3, pp. 190-203, July 2010.

[6] H.B. Kekre and Tanuja K. Sarode, "Vector Quantized Codebook Optimization using K-Means," International Journal on Computer Science and Engineering, Vol. 1, No.3, pp. 283-290, 2009.

[7] S. Vimala, "Convergence Analysis of Codebook Generation Techniques for Vector Quantization using K-Means Clustering Technique," International Journal of Computer Applications, Vol. 21, No. 8, pp. 16-23, May 2011.

[8] J. B. MacQueen, "Some Methods for Classification and Analysis of Multivariate Observations", Proceedings of 5-th Berkeley symposium on Mathematical Statistics and Probability”, Berkely, University of California Press, vol 1, pp. $281-297,1967$.

[9] Y. Linde, A. Buzo, and R. M. Gray, “An algorithm for vector quantizer design,” IEEE Trans. Commun.', Vol. COM-28, No. 1, pp. 84-95, January 1980.

[10] H.B.Kekre and Tanuja K. Sarode, "Two-level Vector Quantization Method for Codebook Generation using Kekre's Proportionate Error Algorithm," International Journal of Image Processing, Vol. 4, No. 1, pp. 1-11, 2010.

[11] H.B.Kekre and Tanuja K. Sarode, "Fast Codebook Search Algorithm for Vector Quantization using Sorting Technique," International Conference on Advances in Computing, Communication and Control, pp. 317-325, 2009.

[12] Tanuja K. Sarode and Nabanita Mandal, "K-Means Codebook Optimization using KFCG Clustering Technique," International Journal of Computer Applications, Vol. 78, No. 6, pp. 38-43, September 2013. 